\title{
Quantum Cascade Laser Enabled Nano-liter Polymer Waveguide Sensor
}

\author{
Sheng Wu, Andrei Deev \\ PEER Institute \\ 738 Arrow Grand Circle, Covina, CA 91722 \\ California Institute of Technology \\ 139-74 Caltech, Pasadena, CA 91125
}

\begin{abstract}
To improve the Mid-Infrared (IR) chemical sensing capabilities in liquids and gases, a polymer based waveguide that has 100\% interaction with Quantum Cascade (QC) laser field is proposed and demonstrated. The waveguide has thickness down to 10 s nanometers so that chemical diffusion and preconcentration could happen very fast; the path length is increased from several microns to over centimeters due to the high spectral and diffraction brightness of QC lasers. Efficient prism coupling into whispering gallery resonators' coated with submicron polymers and planar slab polymer waveguide are demonstrated, high signal to noise ratio is obtained and potential applications discussed.
\end{abstract}

\section{KEY WORD LIST}

Green Photonics, Quantum Cascade laser; Polymer Waveguide sensor, Gas Chromatography, Liquid Chromatography

\section{INTRODUCTION}

\section{Reviving Infrared detector for Gas Chromatography with Quantum Cascade Lasers}

Half a century ago, Infrared (IR) spectroscopy was used much more often than Mass Spectrometry (MS) as a spectroscopic method to qualify and quantify chemicals during chemical analysis processes. This held true even for early chromatography scientists, due to the fact that at that time modern scanning MS was not developed and IR spectroscopy based on dispersive blackbody IR spectroscopy was relatively affordable to a lot of laboratories. The invention of FTIR instruments in the 70's further improved the speed and sensitivity of IR spectroscopy, so even after the success of scanning MS in the late 60's and early 70's, when the leading Chromatography instrument company Agilent, then HP, introduced capillary GC and bench top detector MSD (Mass Spectrometry Detector) just 35 years ago, they also introduced another detector that could uniquely identify the chemical elute peaks and it was based on Infrared detection (IRD) using FTIR as the spectroscopic identification tool. Over the decades, HP/Agilent along with other vendors sold hundreds of thousands of GC-MSDs, but the GC-IRDs are in the lower 100s. The reason behind this sales/market disparity is the poor sensitivity of IRD. Infrared detection is not necessarily a method associated with poor sensitivity, given the fact that molecules often have very strong absorption in the Mid-IR. The bottleneck was that for blackbody sources used in FTIR, the light sources could not be efficiently coupled into capillary IR light waveguide, resulting in very short optical path, and also enlarged bore size and accompanying dilution by makeup gases further degrade the performance of IRD[1]. With the advent and advances of Quantum Cascade lasers, we demonstrated that very high coupling efficiency for QC lasers into IR hollow waveguide (HWG) could be realized[2]. This effectively increased the pathlength of optical absorption to meters from inches, and with no sacrifice in markup gas dilution because the possibility to use small bore HWG. The fast and wide scanning, as well as high spectral purity capabilities of QC lasers[3, 4] [5] further promise to improve the performance of GC-IRD close to that of GCMSD. Now, with powerful QC lasers GC-IRD could have a revival, yet there remains much great challenge if we want to apply IRD to Liquid Chromatography (LC).

Quantum Sensing and Nanophotonic Devices IX, edited by Manijeh Razeghi, Eric Tournie, Gail J. Brown, Proc. of SPIE Vol. 8268, 82680D - (c) 2012 SPIE · CCC code: 0277-786X/12/\$18 - doi: 10.1117/12.905899 
The key parameter we need to mention for modern high performance chromatography instruments, in particularly GC and LC, is the volume of the samples coming out of the chromatography is quite small, i.e. usually $10 \mathrm{~s}$ of micro liters. Therefore, detectors should ideally need zero sample volume, or nanoliters of sample is enough.

For Liquid Chromatography (LC), the chemicals in liquid is usually a lot denser than chemicals in gas at the same concentration level (weight for liquid and by volume for gas), i.e. the amount of chemicals in 1 gram (or $1 \mathrm{ml}$ ) of water is about 1,000 times more than the amount of chemicals in $1 \mathrm{ml}$ of air. Thus, to measure the same concentration of chemical, the path length could be 1,000 times smaller, i.e. from several meters in gas phase to several millimeters in liquid phase. Griffith writes in the $2^{\text {nd }}$ edition of "Fourier Transform Infra Red Spectrometer" ([6], page 495)--- "the path length of a solution that contains $1 \mathrm{ppm}$ of a trace component must be $1 \mathrm{~cm}$ if the solute is to yield a recognizable spectrum"

However, the absorption of liquid, e.g. the prevalent water, in the Mid-IR is very strong, usually less than $0.1 \%$ MidIR photon is left after just $10 \mu \mathrm{m}$ of water layer (yes $30 \mathrm{~dB}$ loss in $10 \mathrm{micron!})$. Organic solvent, e.g. cyclohexane, might have less than $1 \mathrm{~mm}$ path length in thickness before $99 \%$ of Mid-IR photon is absorbe $(20 \mathrm{~dB} / \mathrm{mm})$ to generate reasonable path length of a couple of millimeters, while water is the most often used carrier phase in LC. This means that direct measurement inside water is very hard, if not possible.

In order to measure the chemicals in the liquid phase over relatively long path length, i.e. we need to separate the chemicals from water phase, at least temporarily for the measurement to finish. This strategy is used by several past reports already, i.e. a layer of water repelling polymer will keep water out while adsorbs chemicals of interest for sensing.

Several reports demonstrated coupling QC laser along with FTIR source into solid waveguides and performed many FTIR-ATR (Attenuated Total Reflection prism where many reflections happens inside) experiments. All the past reported experiments [7-9]in the Mid-IR, which uses coated polymer as the water repulsion and chemical enrichment layer, seem to stop short of using single mode waveguide based evanescent wave sensing which probably is not readily available in the Mid-IR. There is also no reported work that measures chemicals in the polymer coating layer directly without using evanescent wave, except the work using slotted Photonic Crystal slow light waveguide in the near-IR[10]. So the effective absorption path length for the chemical of interest is greatly reduced even though waveguide could deliver several millimeter long physical pathlength.

As shown in figure 1, the chemicals inside the coating right on the outside of solid waveguide only interacts with much LESS than 1\% of the light traveling inside the solid waveguide, and it also takes much longer time for the chemicals to travel several microns to reach the solid waveguide surface.

Polymers have been long recognized as an alternative sample preparation and spectroscopy platform for IR sensing and chemical analysis[11], for its capabilities in repelling water while preferentially adsorbing and pre-concentrating chemicals of interest, in both liquid and gas phases[12]. However, when only used in surface evanescent wave sensing scheme, the effective absorption path length is greatly reduced to 10s of microns; the simple transmission type of IR absorption scheme could have longer path length, but it is also compromised due to the much longer time required for chemicals to diffuse into the thick polymers.

The thickness of the waveguide should be minimized to 10 microns or less for Mid-IR wave in the polymers; the thinner the thickness the faster the adsorption and desorption process for the polymers when interact with outside chemicals. When the thickness is reduced to a couple microns, the response time could be as short as a couple of seconds[13]. 
Why only QC laser shines in the thin polymer waveguide?

Regular Mid-IR light source could not be coupled into the ultra-thin $(<<10 \mu \mathrm{m})$ polymer waveguide and travel over even $0.1 \mathrm{~mm}$ before all leaking out. Only QC laser which has close to diffraction limit brightness could be coupled efficiently into and travel several millimeters inside the thin waveguide.

\section{Designing the Mid-IR Single Mode Polymer Waveguide (MIR-SMPW) as a sensor platform}

The major challenge for designing the MIR-SMPW as a sensor platform is to confine a big portion of the Mid-IR lightwave inside the polymer waveguide, instead of left out in the substrate or the strongly attenuating solvent outside. The thickness should also be as thin as possible to facilitate faster equilibrium time constant. The refractive index of polymers of interest in the Mid-IR is usually around $<1.4$ and 1.6, i.e. slightly larger than liquid and much smaller than many Chalcogenide glass materials. This means that for planar SMPW, the substrates must be CaF2 or BaF2, not with refractive index higher than the polymer, otherwise the single mode waveguide is no longer valid. For SMPW inside WGR, the WGR cavity refractive index is no longer important because the Mid-IR light wave is forced to circulate on the most outside waveguide due to geometric factors.

\section{Polymer thin film as substrate for Mid-IR sensing}

Transparency of polymer thin films in the Mid-IR

Figures 2 show that where there is no vibrational absorption peaks the polymers have relatively wide transparent regions where the path length for chemical sensing could be several millimeters. By using deuterated polyethylene, the transparency region could also be engineered (see DPE IR spectrum in figure 3). The typical absorption in the transparent region of Polyethylene (PE) is about $50 \% / \mathrm{mm}$, or $30 \mathrm{~dB} / \mathrm{cm}$. This means that with powerful quantum cascade lasers, we should be able to realize path lengths over $10 \mathrm{~mm}$ in a wide range of the Mid-IR, and measure chemicals adsorbed in polymers with an accuracy of 1ppm or better if the absorption feature is relatively strong. Polymers also could be engineered to have enrichment capability to chemicals therefore improve the detectivity.

\section{EXPERIMENT}

\section{Transmission parameters inside}

The schematic for fast and wide frequency tuning for DFB QC lasers is given in figure 1. The laser output is collimated and coupled into a WGM cavity with $0.47 \mathrm{~cm}-1$ Free-Spectra Range (FSR), and by counting the period in each current ramping cycle, we could calculate the frequency tuning range as well as mode purity or mode hopping.

\section{Fabrication of Mid-IR Single Mode Polymer Waveguide}

We dissolve the polymer of interest in Toluene at elevated temperature of $70^{\circ} \mathrm{C}$, and then after it cools down use only the top clear solution as coating solution. We dip the CaF2 WGR ball inside the clear polymer solution and then let the Toluene solvent dry up, and then conduct the characterization process similar to the way we couple QC laser into the CaF2 WGR to characterize its modes. Here, two prisms are used to couple the light in and out of the CaF2 WGR. To show that we achieved nanometer polymer coating on the CaF2 WGR, we used a flat KRS/CaF2 window as a witness sample and dip it in the same PolyEthylene solution, and then measure the FTIR spectra after Toluene dries up. Figure $3 \mathrm{a} \sim 3 \mathrm{c}$ show the results of the coated $\mathrm{PE}$ film on $\mathrm{KRS} / \mathrm{CaF} 2$ window substrate has a peak absorption of only 0.003 at $3,000 \mathrm{~cm}-1$, whereas a 10 micron thick PE film (clingwrap) has an absorption of 3 . This translates into a PE coating thickness of $10 \mathrm{~nm}$ ! This dip-dry-measurement process could be repeated several times to increase the PE coating thickness with quite repeatable increment steps. This created our first CaF2 WGR single mode waveguide for polymers with different thickness. 
The second single mode polymer waveguide is created by dipping the $\mathrm{CaF} 2$ window inside hot PE toluene solutions, and repeat the dip-dry-measurement process several time, with each time adding about 100nm of PE. We decide the final thickness of PE film by measuring the FTIR spectra.

\section{Coupling QC lasers into MIR-SMPW}

We coupled QC laser into the MIR-SMPW with two methods. The first approach is a simple method due to the fact that we only coat the outside of the whispering gallery resonator (WGR) with a thin layer of polymer, and therefore Mid-IR laser only travels the outside solid part the of WGR, and Mid-IR lightwave SMPW is automatically demonstrated when we couple the QC laser into and out of the CaF2 WGR with two prisms, as we demonstrated before for polished CaF2 WGR balls without polymer coatings[14, 15].

The $2^{\text {nd }}$ approach is to use prisms (Rutile prism from Metricom) with larger refractive index than the planar waveguide polymer and couple QC laser into the planar waveguide through the first prism and after travel a couple of millimeters coupled out from the $2^{\text {nd }}$ prism.

\section{RESULTS}

Table 1 gives the absorption or transmission data of PE polymer when probed by a QC laser at 4.4microns. The unattenuated QC laser will saturate the detector, and scaled signal is over 30 Volts. After passing through several PolyEthylene/PolyPropylene (High Density PolyEthylene, or HDPE; and also Low Density PE, or LDPE; and PolyPropylene, or PP), Plexiglass, COC (cyclic olefin copolymer) and PTFE (PolyTetraFluoroEthylene), we get different signal levels. This transmission data shows that with powerful QC lasers, the effective pathlength is increased to several millimeters easily inside the polymers while still have several volt of signal and the noise level of the detector (TEC cooled) is just on the order of $\sim 2$ milli-volt scale. By average over 1,000 scans, we could have a noise level below $0.2 \mathrm{mV}$, and therefore achieve signal to noise ratio over 1,000 .

So for HDPE at $4.45 \mu \mathrm{m}$, the loss is about $\sim 40 \mathrm{~dB} / \mathrm{cm}$; for LDPE it is about $\sim 46 \mathrm{~dB} / \mathrm{cm}$; and for PP it is about $60 \mathrm{~dB} / \mathrm{cm}$ and COC has similar number as PP.

Figure 4 shows FTIR absorption spectra of 1.6mm samples of LDPE, HDPE and PP. When compared to organic solvent and water absorption spectra[16], PE Polymer has the lowest absorption coefficient when the wavelength is not in the absorption peak spectrum region. If using cyclohexane as the extraction and detection solvent at $1,400 \mathrm{~cm}^{-1}$, the pathlength will be limited to just around $0.5 \mathrm{~mm}$ with about $20 \mathrm{~dB}$ attenuation; while for LDPE, the pathlength could be $2 \mathrm{~mm}$ and for HDPE the pathlength could be $4 \mathrm{~mm}$.

\section{DISCUSSIONS}

\section{Effective absorption pathlength for Mid-IR Single Mode Polymer Waveguide (MIR-SMPW) sensor}

If majority of the Mid-IR lightwave resides inside the waveguide's polymer part, then the pathlength is purely limited by the transmission of polymer waveguide in the Mid-IR wavelength range of interest. For good IR transmission polymers, e.g. PE and PP, and at Mid-IR wavelength range where there is no major absorption of the polymer, the pathlength could be up to 10 millimeters if the Mid-IR lightwave could tolerate a $30 \mathrm{~dB}$ loss. At the isolated absorption features Mid-IR wavelength region of these polymers, e.g. 3.3 and 10 microns, one could not make measurements and deuterated polymer could be a good alternative.

\section{Application scenarios of MIR-SMPW sensors}

The first application scenario for the MIR-SMPW sensors is in the liquid phase, e.g. in both bulk liquid phase and as an online sensor for liquid chromatography, or other condensed phase chromatography, e.g. capillary column 
chromatography. In such scenarios, the thickness of the SMPW has to be over a couple of microns in order to confine the majority of the Mid-IR lightwave inside the relatively transparent SMPW, and avoid severe attenuation by the external solvent. This might slow down the response time due to the longer adsorption/desorption process in the relatively thick polymers, but still time constant on the order of less than a couple of seconds is expected when equilibrium is reached, and it could be even faster if equilibrium is not required for maximizing the sensitivity or if the SMPW is chemically tuned for faster adsorption/desorption.

The second application scenario for the MIR-SMPW sensors is in the gas phase, i.e. in a way similar to Solid-Phase Micro Extraction (SPME). Here, the SMPW will not only act the same way as the polymer needles in SPME, but it will also act as the sensor platform when coupled with QC lasers and detectors. When used as a detector, the SMPW could have a very thin layer of polymer, e.g. 10s of nanometers, in order to quickly reach the equilibrium with the gas phase; this ultrathin SMPW is also to minimize the absorption loss due to the polymers and extend the pathlength of the SMPW to over 10s of millimeters. The adsorption and desorption process could be externally controlled for certain chemicals of interest by temperature cycling, which is similar to a temperature programmed GC. We could imagine that tunable QC laser could be tuned to certain chemical's absorption band and eject certain chemicals from the SMPW, and then detect the others with a weaker probe QC laser.

\section{CONCLUSION}

Here, we first investigated the Mid-IR transmission properties of polymers, i.e. PolyEthylene and PolyPropylene. We also demonstrated the generation of nanometer-thin coating of such polymers over optical substrate and form waveguide. The interaction of Mid-IR lightwave and the polymer waveguide is discussed. We coupled Quantum Cascade (QC) laser into a CaF2 Whispering Gallery Resonator with a $40 \mathrm{~nm}$ thick layer of polyethylene coating. Also with prism coupling, we coupled QC laser into a single mode Mid-IR slab polymer waveguide with $\sim 10$ microns thickness. Due to high brightness, only QC lasers could be efficiently coupled into these two waveguides with good signal to noise ratio. These ultra thin polymer waveguides minimizes the time of adsorption to second level while providing preconcentration and water repelling, and they have effective path lengths well over several millimeters for QC lasers. Thus they could realize better than $10^{-3} \mathrm{~Hz}^{1 / 2}$ of loss accuracy in the Mid-IR absorption measurements, i.e. sub ppm level of samples in nano-liters of volume could be detected and identified by QC lasers. The researches here provide a method for conducting sensitive IR detection with liquid chromatography, as well in open air/liquid environment.

Table 1. Signal of QC laser after different polymers at different thickness/pathlength. The scaled signal voltage before passing through the polymers is about 25 Volts. The detector saturated at about 5 Volts.

\begin{tabular}{|c|c|c|c|c|c|c|c|c|c|}
\hline $\begin{array}{l}1 \mathrm{x} \\
1.63 \mathrm{~mm} \\
\text { HDPE }\end{array}$ & $\begin{array}{l}2 \mathrm{x} \\
1.63 \mathrm{~mm} \\
\text { HDPE }\end{array}$ & $\begin{array}{l}3 \mathrm{x} \\
1.63 \mathrm{~mm} \\
\text { HDPE }\end{array}$ & $\begin{array}{l}4 \mathrm{x} \\
1.63 \mathrm{~mm} \\
\mathrm{HDPE}\end{array}$ & $\begin{array}{l}2 \mathrm{x} \\
1.63 \mathrm{~mm} \\
\text { HDPE } \\
+1.63 \mathrm{~mm} \\
\text { LDPE }\end{array}$ & $\begin{array}{l}1 \mathrm{x} \\
1.63 \mathrm{~mm} \\
\text { HDPE x2 } \\
+1.60 \mathrm{~mm} \\
\text { PP }\end{array}$ & $\begin{array}{l}5.46 \mathrm{~mm} \\
\text { clear } \\
\text { Plexiglas } \\
\mathrm{s}\end{array}$ & $\begin{array}{l}1.63 \mathrm{~mm} \\
\text { HDPE x2 } \\
+ \text { COC } \\
5031 \\
1.20 \mathrm{~mm}\end{array}$ & $\begin{array}{l}3.18 \mathrm{~mm} \\
\text { LDPEx2 }\end{array}$ & $\begin{array}{l}3.3 \mathrm{~mm} \\
\text { PTFE }\end{array}$ \\
\hline $\begin{array}{l}\text { saturate } \\
\text { d }\end{array}$ & $\begin{array}{l}\text { saturate } \\
\text { d }\end{array}$ & $2.70 \mathrm{~V}$ & $0.53 \mathrm{~V}$ & $2.50 \mathrm{~V}$ & $1.15 \mathrm{~V}$ & $\begin{array}{l}0.04 \mathrm{~V} \\
\text { after } 100 \\
\text { averages }\end{array}$ & $1.64 \mathrm{~V}$ & $300 \mathrm{mV}$ & $30 \mathrm{mV}$ \\
\hline
\end{tabular}




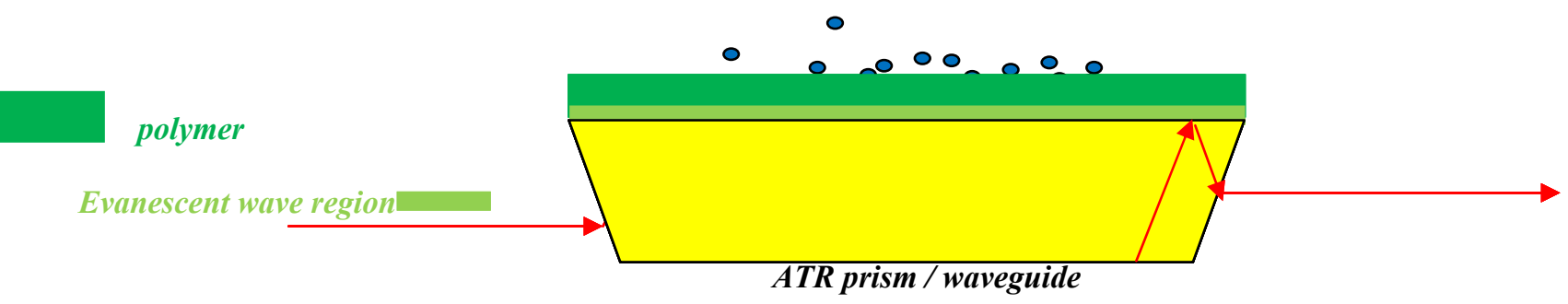

Figure 1. Schematic of traditional evanescent wave sensing. The total effective pathlength is limited to 100s of microns even several dozens of total internal reflection happens. Also, the Mid-IR lightwave is only interacting with a small portion of the adsorbed chemicals if the coating is more than a couple of micron thick.



Figure 2. FTIR spectra of PolyEthylene (PE) and Deuterated PE which shows that the $3,000 \mathrm{~cm}^{-1}, 1450 \mathrm{~cm}^{-1}$ and $650 \mathrm{~cm}^{-1}$ region could be opened up for SMPW sensor. Figure is generated by combining two FTIR spectra of PE and DPE found on the web. 


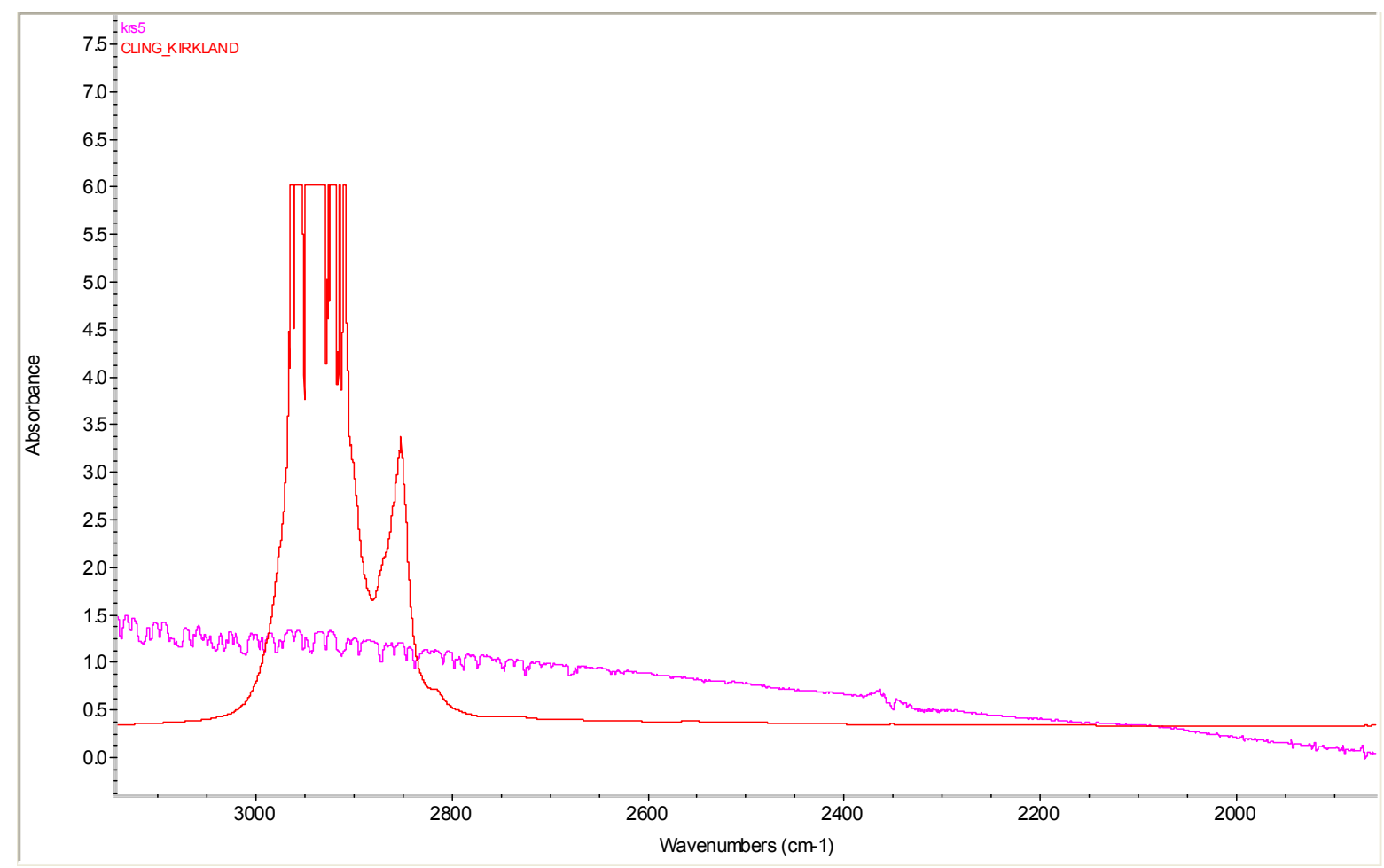

Figure 3a. 10 micron Polyethylene film (red) and KRS substrate (violet) transmission spectra

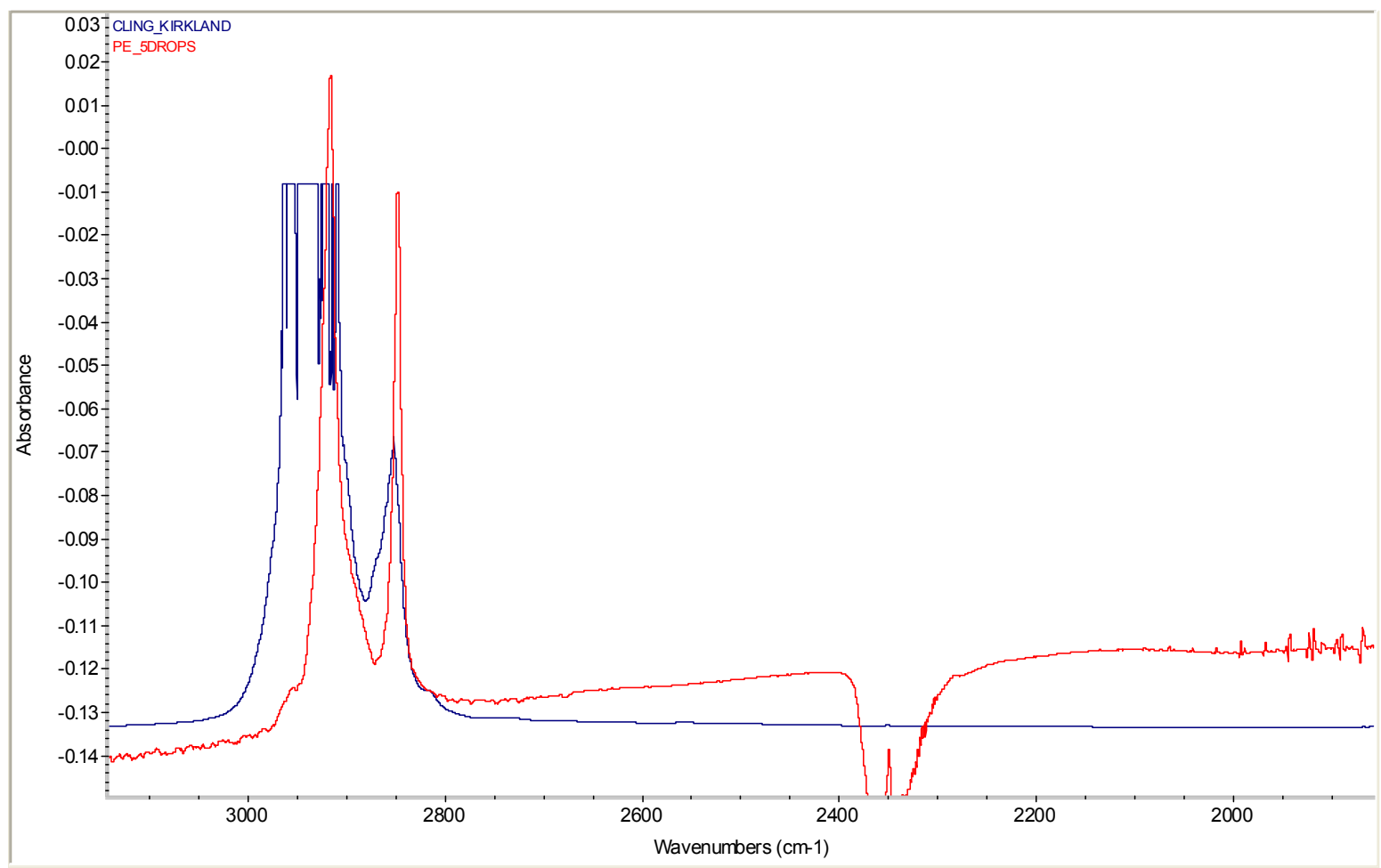

Figure 3b. 5 Dip-Dry steps of PE solution on KRS susbtrate (red) and background is 10 micron thick PE film transmission spectra. The spikes at $2,300 \mathrm{~cm}-1$ is due to $\mathrm{CO} 2$ absorption variations in the air. 


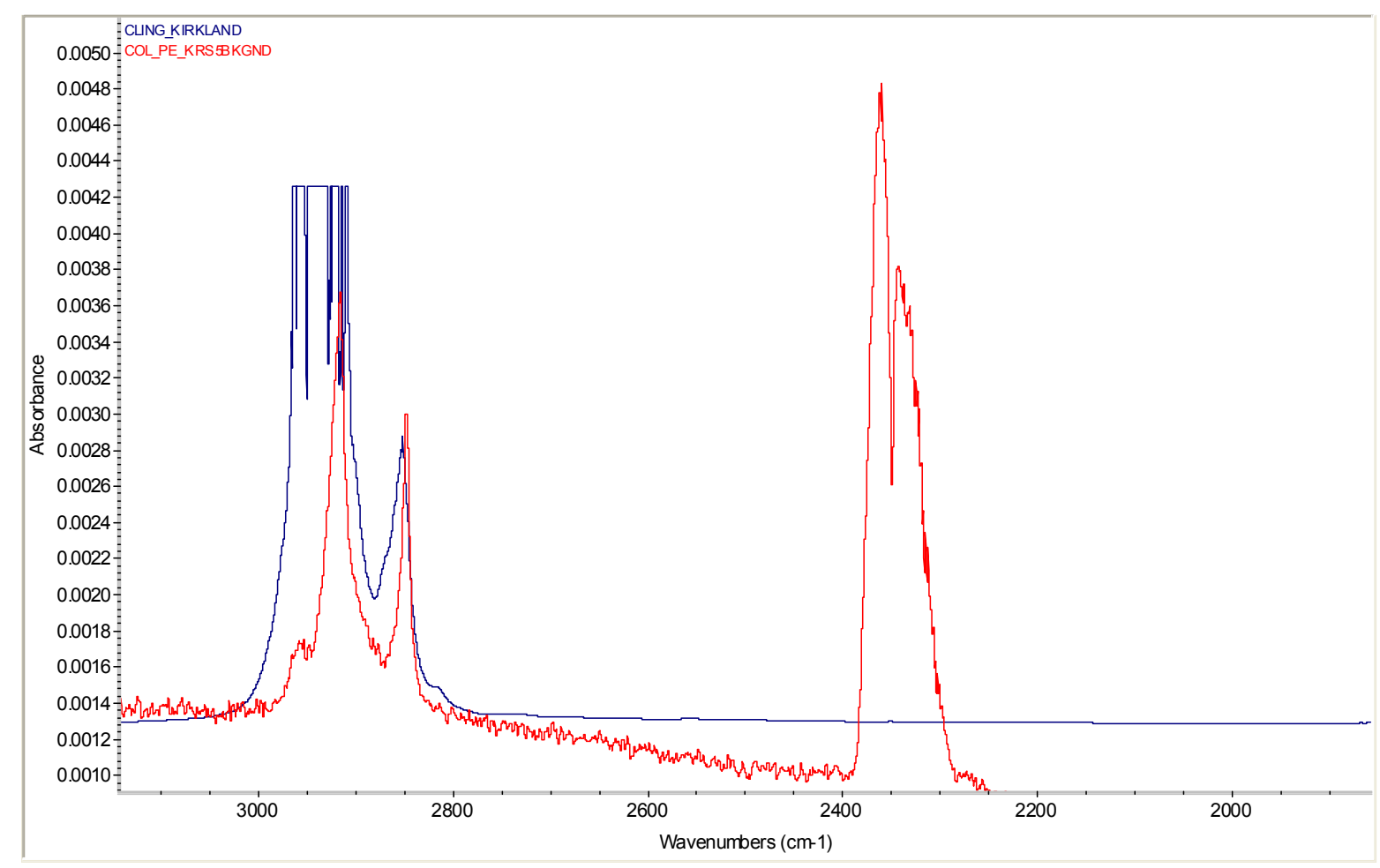

Figure 3c. 2 Dip-Dry steps of Cold PE solution on KRS substrate showing absorption of 0.002 versus 3 recorded with 10 micron thick PE film. This translates into a coated PE film thickness less than $10 \mathrm{~nm}$.

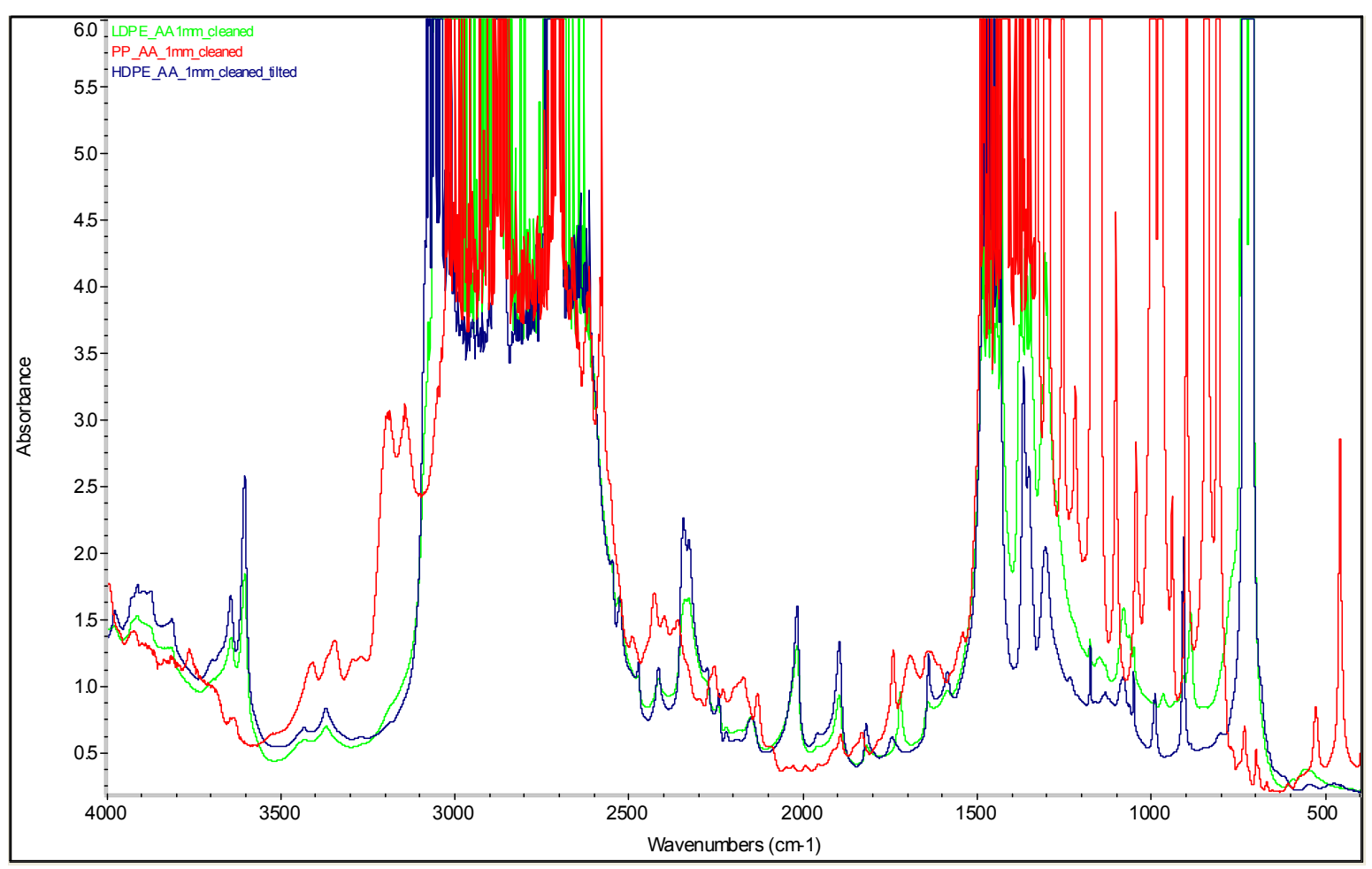

Figure 4. FTIR spectra of 1.6mm thick PE/PP polymers showing the transparency region's regular absorption. 